\author{
Marco Fontana \\ Assistant Professor \\ m.fontana@sssup.it \\ Salsedo Fabio \\ Senior Researcher \\ f.salsedo@sssup.it \\ Simone Marcheschi \\ Research Fellow \\ s.marcheschi@sssup.it \\ Massimo \\ Bergamasco \\ Full Professor \\ m.bergamasco@sssup.it
}

\title{
Haptic Hand Exoskeleton for Precision Grasp Simulation
}

\begin{abstract}
This paper outlines the design and the development of a novel robotic hand exoskeleton conceived for haptic interaction in the context of virtual reality and teleoperation applications. The device allows exerting controlled forces on fingertips of the index and thumb of the operator. The new exoskeleton features several design solutions adopted with the aim of optimizing force accuracy and resolution. The use of remote centers of motion mechanisms allows achieving a compact and lightweight design. An improved stiffness of the transmission and reduced requirements for the electromechanical actuators are obtained thanks to a novel principle for integrating speed reduction into torque transmission systems. A custom designed force sensor and integrated electronics are employed to further improve performances. The electro-mechanical design of the device and the experimental characterization are presented.
\end{abstract}

\section{Introduction}

A Hand Exoskeleton (HE) is a robotic device whose mechanical structure is located in close proximity of fingers of human hands. The kinematics of such a kind of device is often designed in a way that linkages follow the movements of the human operator. HEs have attracted a lot of attention in haptics and robotics research communities thanks to their many advantages and good properties. Their basic functionality consists in exerting programmable forces on the human fingers. Their most relevant feature is the optimal matching between the human reachable workspace and robot workspace. Moreover, HEs are preferred over desktop haptic interfaces for applications where large workspace and reduced visual/spatial encumbrance is needed.

HEs can be classified in two functional categories depending on the capability of exerting forces on one phalanx only or on multiple phalanges. Multi-Phalanx Hand Exoskeletons (MPHE) are devices able to exert different forces on at least two of the phalanges of the same finger (see Fig. 1-left); commonly the force can be applied on a fixed direction normal to the phalanx axis and belonging to the medial plane of the finger. Single-Phalanx Hand Exoskeletons (SPHE) are able to exert forces on one phalanx of the finger, usually the distal phalanx only (fingertip); some devices are able to generate force only along a fixed direction but more commonly they can exert forces along any wanted direction (see Fig.1-right).

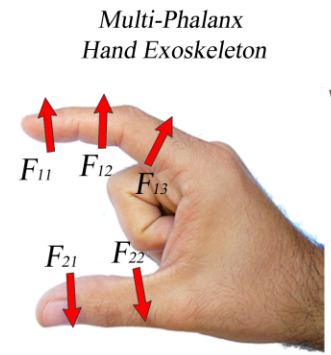

Forces along fixed directions
Single-Phalanx

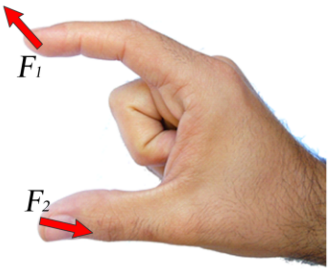

Forces along any wanted direction
Hand Exoskeleton

Fig. 1. Multi-Phalanx and Single-Phalanx scheme
HE can be further distinguished in two other categories:

- Anthropomorphic: the kinematic of the HE is morphologically similar to the kinematic of the human fingers;

- Non-anthropomorphic: the kinematic of the HE is morphologically different from the finger kinematic scheme.

In the scientific literature of the last 30 years, there are many works reporting the design of different HEs. The different devices can be also classified on the basis of the application field as follows:

- Virtual Reality (VR) or Tele-Operation (TO): HEs are used as a haptic interface in VR application for the generation of illusory forces or as master controller that provide force feedback in TO systems. In both applications the HE assumes similar functional aspects.

- Medical applications: HEs are employed in rehabilitation therapy or as active orthosis for restoring hand capabilities after strokes or accidents.

- Space applications: HEs are employed as an active device as a force extender for helping the astronauts in Extra Vehicular Activities (EVA).

The first known example of HE was introduced in the field of teleoperation by Zarudiansky back in 1981 [1]. The inventor registered a patent on a tele-manipulation system equipped with a master device able to provide force feedback on different phalanges of human fingers. At JPL laboratory, in 1988, Jau [2] built a complete teleoperated master/slave arm comprehending a four-finger HE. In the same years Burdea developed a pneumatic actuated hand force feedback device [3], called Rutger Master, able to exert forces on the fingertip along a direction that is assigned by the kinematic of the device and the position of the finger. In the same years, Bergamasco et al. [4], at PERCRO Laboratory, developed another four-finger MPHE able to exert forces on each phalanx of fingers. A non-anthropomorphic device was realized by Koyama from Keio University. This device is a three-finger exoskeleton for thumb, index and middle finger with passive clutches actuation [5]. Such HE is anchored on the wrist of the user and it is able to exert forces in every direction belonging to the sagittal plane of the fingers. Frisoli [6] developed 
another non-anthropomorphic HE called Pure Form Hand-Exos for VR applications that features a complex steel cable transmission studied for an optimal use of motor torque. Nakagawara [7] built a device integrating the concept of encountered haptics in the field of exoskeletons. The device has one degree of freedom for each finger and it is able to track the finger of the user without any contact (even at the fingertip) through a non-contact sensor placed in correspondence of the nail. When the contact takes place in the remote environment a plate is moved against the user fingertip.

Other examples of HEs have been developed and employed in medical applications. In this field the HE are employed in rehabilitation procedures or as power assisting device (orthesis). Gomez [8] has integrated the Rutger Master in a virtual reality rehabilitation system. Wege [9] develop a 4 Degrees of Freedom (DoF) HE with a modular structure, actuated with Bowden cables. Mulas [10] realize an HE that makes use of EMG signals for predicting the movements of the user finger. Ito [11] built a complex HE for rehabilitation purposes with 4 DoF for the index, medium and ring finger and $5 \mathrm{DoF}$ for the thumb able to exert controlled torque on each of the phalanx. The first HE conceived as an orthosis for the finger was developed by Brown [12]. The realized device was an HE composed by three rings attached on the phalanges that are actuated by pulling cables. Other fingerorthoses have been realized lately, some examples are reported in $[13,14]$.

Another application where HEs have proved their usefulness is in assisting astronauts in performing Extra-Vehicular Activities (EVA). Pressure difference causes stiffening of the astronaut's gloves impeding manipulative tasks. For this reason, custom designed $\mathrm{HE}$ are used as an active tool for reducing the perceived stiffness of the glove. Shields et al. [15] developed the first HE for space applications. The developed device was an anthropomorphic HE with three active fingers. HE has 2-DoFs for each finger and exploits special mechanisms for the implementation of the finger flexion joints. Anthropomorphic kinematic is obtained through remote center of rotation joints. Yamada in 2001 [16] developed another simplified HE called Skilmate for space applications. Only the first flexion DoF of each finger is actuated since it's the most solicited in this kind of application.

As a general consideration, we can observed that HEs realized for VR or TO have several mechanical features that are not common with the devices that are conceived for rehabilitation, orthosis or EVA applications. VR and TO require the simulation of fine haptic interaction and generally more demanding performances are needed in terms of mechanical features. In the design of $\mathrm{HE}$ for $\mathrm{VR}$ and $\mathrm{TO}$ a particular care is given to minimize friction, inertia and weights. In other applications, higher level of friction is tolerable allowing the use of Bowden cables for delocalizing the motors or simplified kinematic structure that allows exerting forces with less accuracy. On the contrary, the aim of the work that is described in this paper is the development of a high performance device featuring high level of force accuracy and resolution.

In the following sections we describe the design procedure of the developed HE able to exert forces in the range of few Newtons but with high capabilities of accuracy and resolution. The target of application of the HE that is described in this paper is the haptic interaction in VR or in TO systems. The reference tasks are the precision grasping between index and thumb, the manipulation and the exploration of surfaces and objects involving forces in the range of $0-5 N$. The realized device (shown in Fig. 2) is able to exert controlled forces along any wanted direction on the two fingertips of the index and thumb of the right hand of the operator. In the second section of the paper we briefly describe the general design guidelines that has driven the design work.

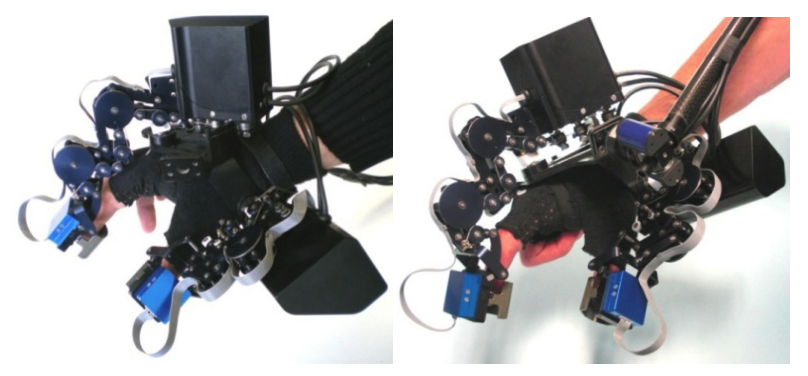

Fig. 2. Picture of the assembly of the realized Hand Exoskeleton without (left) and with tracking system (right).

In the third and fourth sections we describe respectively the kinematics and the actuation systems, focusing on the novel introduced aspects. The fifth section briefly reports the design process of the integrated force sensor that has been purposely designed [17]. The sixth section is dedicated to the description of the custom acquisition and driving electronics and the last section reports the tests and characterization of the whole device.

\section{Design guidelines}

The artificial generation of the forces that are felt when interacting with objects directly using the hands is the final objective of the haptic HE that is described in this work. In particular the target application is the simulation of two types of interaction: (1) Precision Grasp and (2) Surface Exploration. Jones and Lederman in [18] describe Precision Grasp as the action that is done while holding and manipulating an object between the fingertips of thumb and index finger. Surface Exploration is the common action of exploring the surface of an object by stroking different parts with our hands. Both Precision Grasp and Surface Exploration are extremely complex kinds of hand-object interactions. Such kind of manipulative actions involve contact forces whose direction can be generally along three dimensions and whose intensity varies in a very large range, from tens of Newtons of maximum force that can be exerted (during hard grip) [19] to forces in the order of milli-Newtons for the minimum perceivable force when the finger is passively stimulated [20]. However, while low forces (in the range of fraction of Newtons) are quite frequent and important in everyday manipulative actions, the exertion of maximum forces is a rare event. Analysis of force and their statistical distribution in some common Activities of Day Living (ADL) is provided by Redmond et al. in [21]. These authors show that many small lightweight objects like pens, objects on the desk, portable phones, PCs and phone keyboards, etc. are effectively manipulated with forces in the range from fractions of Newtons up to some Newtons. For this reason, many haptic devices have been designed to operate in the force-range of a few Newtons and, accordingly, the target force capability for the present $\mathrm{HE}$ has been set in the same range. In particular a maximum operating force $F_{\max }=5 \mathrm{~N}$ has been chosen. At the same time the aim of the design of the proposed HE has been 
oriented toward the optimization of resolution performances in such a range of forces.

Force resolution of haptic interfaces is usually limited by several mechanical issues. Friction, backlash, compliance and large inertia of the mechanical structure are among the most relevant factors that contribute to the generation of disturbing forces that reduce the global resolution. The choice of an exoskeleton-like device has a positive influence on the quality of force generation since it allows to limit the length/weight/inertia of the linkages, the power of the actuators and the length of transmissions without limiting the reachable workspace. However, the weight of wearable devices must be carried by user and consequently their design have to be optimized in order to minimize masses of mechanical structure and actuators. Alternatively the weight of the wearable device can be relieved by an external weight compensation system [22].

The whole mechatronic design of the presented HE has been oriented toward the primary objective of optimizing force resolution at best and the secondary objective of minimizing the global weight of the device.

In order to obtain optimal force resolution, design has been based on a set of special mechanical solutions that includes: (a) custom mechanisms, (b) lightweight mechanical structures, (c) semiremote lightweight actuators, (d) pre-tensioned steel cable transmission, (e) custom force sensors for implementing active compensation and (f) integrated control and driver electronics.

\section{Kinematics}

The kinematic scheme that has been adopted for the developed HE can be defined as a quasi-anthropomorphic kinematics. The choice was driven by the fact that anthropomorphic kinematics presents many positive features that well fit the requirements of a portable haptic device such as: (a) optimal matching between workspace of the mechanism and required workspace; (b) short length linkages; (c) optimal wearability with limited visual encumbrance.

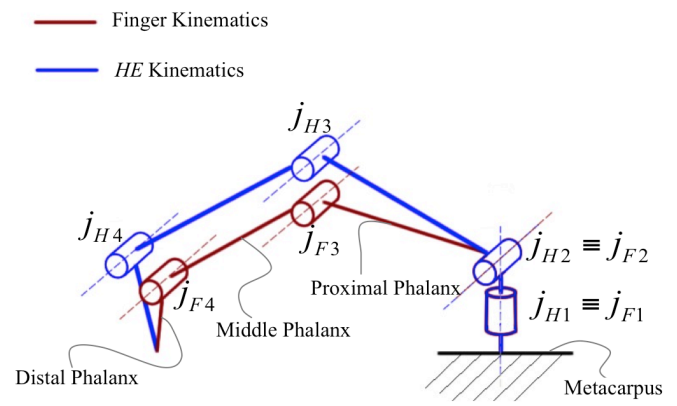

Fig. 3. Scheme of the quasi-anthropomorphic kinematics of the HE. Joints of the human finger are named $j_{F i}$ and joint of the haptic interface are named $\boldsymbol{j}_{\mathrm{Hi}}$. The picture refers to index finger scheme. The scheme for the thumb is analogous.

Anthropomorphic kinematics is usually adopted for MPHE since the links of the device have to accurately follow the movements of the phalanges. Differently, in our design, we adopt a kinematics that is morphologically identical to finger kinematics but it slightly differs for the length of the links. The perfect coincidence would be desirable but it is not allowed to avoid the singularity position when the finger is completely extended. The concept of quasi-anthropomorphic kinematics is represented in Fig.3. The simplified kinematic model of the finger that has been taken as reference is described in by Springer and Ferrier in [23]. Such model considers both the index and thumb finger as $4 \mathrm{R}$ manipulator. A more accurate kinematic model of the thumb finger would consider 5 DoFs as in [24] but for limiting the complexity of the device we accept the trade-off neglecting adabduction of the metacarpophalangeal joint.

A further simplification has been assumed for reducing the number of actuators. The actuation of 4-DoFs would require the use of four actuators and, since the actuators generally constitute the heaviest fraction of the mechanical assembly of a haptic interface, we introduce a simplification that allows the use of only three motors. The rotation between the distal and middle phalanx (joint $j_{H 4}$ ) has been coupled with the rotation between the middle and the proximal phalanx (joint $j_{H 3}$ ). This coupling reflects the coupling that is present in the unconstrained motion of human fingers (as described in [25]). However, while this coupling for human hand's finger follows a complex relation we introduce a simplification assuming a constant ratio between the rotation of the joint $j_{F 4}$ and the rotation of the joint $j_{F 3}$ is constant. This simplification is more critical for the thumb since the coupling between phalanxes is weaker. However, for the sake of compactness and lightweight this has been considered tolerable.

The global dimensions of the mechanism that implements the $\mathrm{HE}$ has been dimensioned referring to average value of anthropometric dimensions of phalanges [26]. In order to reduce complexity and to avoid compromising reliability of the system, no special registrations have been foreseen for adaptation to different anthropometric dimensions. However, the length of the links is approximately $15 \%$ larger respect to the hand average values. This is done since operators with lager hands could have reached the boundary singularity of the mechanism before being able to extend their finger.

\subsection{Adduction-Abduction Joint $\left(j_{H 1}\right)$}

The first joint indicated with $j_{H I}$ in Fig.4 is implemented through a bearing joint whose encumbrance is located on the dorsal side of the hand. The axis has been oriented along the normal direction to the palm of the hand. The axis of $j_{H 1}$ is through the center of rotation of the metacarpophalangeal joint for the index finger and with the carpometacarpal joint for the thumb.

\subsection{Flexion-Extension Joints $\left(\boldsymbol{j}_{H_{2}}\right.$ and $\left.\boldsymbol{j}_{H_{3}}\right)$}

A critical aspect of the implementation of a quasianthropomorphic exoskeleton is the design of the flexionextension joints $j_{H 2}$ and $j_{H 3}$. The layout of such joints has to be chosen considering that collisions may occur between the links and phalanges. A possible solution consists in using bearing joints at the sides of the finger as proposed in [27]. In such solution, the joint's bearings are located on both the sides of the finger. This implementation is functional and reliable but introduces limitation given by the lateral encumbrance of the joints that impede the fingers to come close each other. The solution that we adopted makes use of Remote Center of Motion (RCM). RCM is a mechanism that is able to implement the rotation of a body around an axis that is remotely located from the structure of the joint. There are several mechanisms able to implement this feature. Many of them are reported and analyzed by Pei [28] and Fiaschi [29]. Fiaschi has analyzed several RCMs for the implementation 
of flexion joints of HEs. The analysis consists of a score ranking that considers the complexity of implementation, the encumbrance and the mechanical characteristics. The RCM, represented in Fig.4 for both the flexion joints, composed by two parallelograms connected together to form a 6-bar mechanism, resulted the best ranked in this analysis. The 6-bar RCM mechanism allows the $\mathrm{Li}$ to rotate around the Remote Center of Rotation (RCR) that is an axis of rotation located remotely respect to the encumbrance of the mechanisms. Such RCM has been chosen for the implementation of the flexion joints of the developed HE allowing to locate mechanism on the dorsal side of the finger.

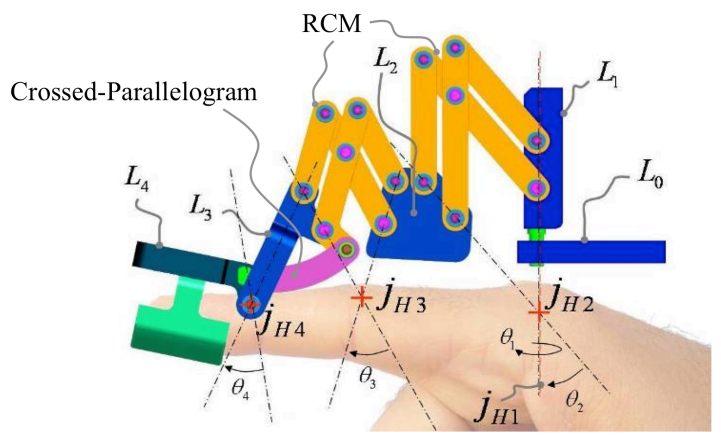

Fig. 4. Implementation of the finger kinematics including the Remote Center of Motion mechanisms

\subsection{Distal flexion Joint $\left(j_{H_{4}}\right)$}

The implementation of the distal joint $j_{H 4}$ could have been analogous to the joints $j_{H_{2}}$ and $j_{H 3}$, but the choice was to use lateral bearings solution. The choice was commanded by the need of leaving the dorsal side of the last link free to host a tactile array that is described in [30].

As previously mentioned the rotation of the joint $j_{H 4}$ is coupled with the rotation of the joint $j_{H 3}$. The coupling between the joints $j_{H 4}$ and $j_{H 3}$ has been implemented with a crossed parallelogram represented in Fig.4. The angle $\theta_{3}$, that sets the rotation between the links $L_{3}$ and $L_{2}$, is taken as input rotation for the crossed parallelogram mechanism. The output angle $\theta_{4}$ is function of the input angle with a ratio that varies from 0.8-0.9 in the used angular range. The details of the dimensioning of such mechanism are explained in [31].

\section{Force transmission and actuation}

The weight of the actuators usually represents the largest fraction of the global weight of an HE thus their arrangement has to be carefully considered. A simple solution, for devices that are based on a serial kinematics scheme, is to position the actuators directly on the joint axes. Unfortunately, this solution is hardly usable when high performances are required since it leads the structure to show high inertia and there is a high waste of usable motor-torque for gravity-compensation. An alternative approach is to delocalize the motors from the linkage structure and positioning them on the base of the mechanism using steel cable for transmitting their torques to the joints. This solution guarantees low friction and backlash and has been chosen for many haptic interface, but the design is much more complex and the manufacturing can be quite expensive. In our design, we adopted a partial delocalization of actuators. The motors have been positioned on the first link $L_{l}$ of the mechanism as represented in
Fig.5. This solution allows, at the same time, to avoid the large moving masses and to introduce a routing of the actuation cables for the joints $j_{H 2}$ and $j_{H 3}$ characterized by a reasonable level of complexity.

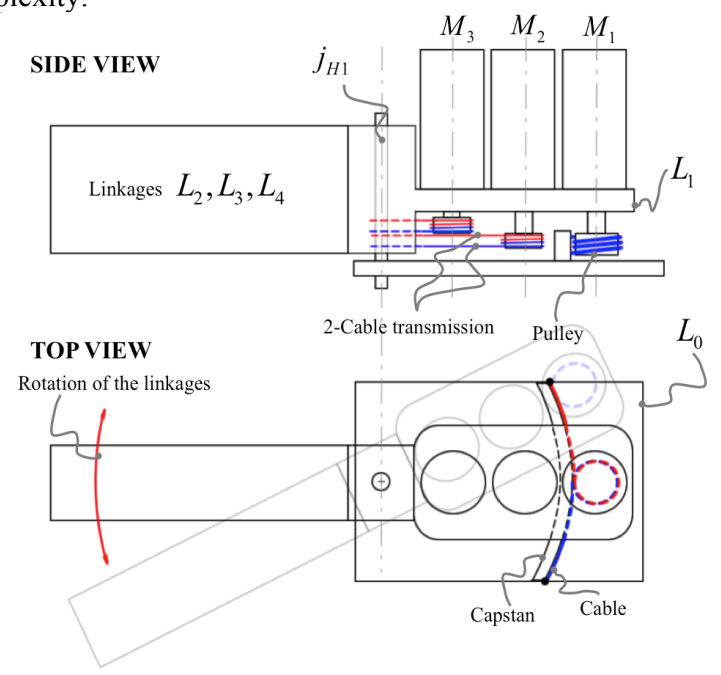

Fig. 5. Motor location and routing of transmission for the joint jH1

The joint $j_{H I}$ is actuated through a capstan-pulley transmission (represented in Fig. 5). This implementation includes the use of a double cable transmission, i.e. a cable for driving the abduction and a different cable for the adduction. Speed reduction ratio is $r_{11}=R_{m 1} / R_{c}$, where $R_{m 1}$ and $R_{c}$ are the radius of motor and capstan pulleys. A different solution has been considered, for the actuation of the RCM that implements flexion joints. The simple use of a capstan-pulley transmission could be applicable also in this case (see Fig. 6), but it would require large radius for the driven pulley $\left(R_{l}\right)$ in order for the speed reduction to reach a significant value. A novel method for actuating the RCM has been conceived and patented [32]. The idea is based on exploiting the mutual rotation of the various links of the 6-bar mechanism in order to multiply the length-change of the cable as the joint rotates. The implementation of this idea is achieved adding idle pulleys that are coaxial-disposed with the joint-axes of the RCM and arranging the cables along a path from the motor pulley, around the different idle pulleys and closing its end attachment on one of the links.

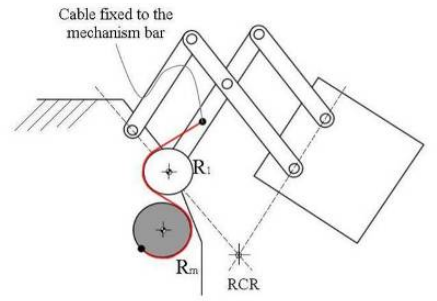

Fig. 6. Possible scheme of capstan-pulley transmission for the RCM using one idle pulley.

Some possible variants of such kind of transmission system are represented in Fig. 7. It can be shown that this transmission system leads to an intrinsic amplification of speed reduction ratio. Speed reduction can be computed calculating the rotation of the 
motor axis for a unit rotation of the joint. In particular, when the joint rotates, the variation of length of the cable path is equal the sum of the changes of length of each cable segment that is wrapped around each pulley.
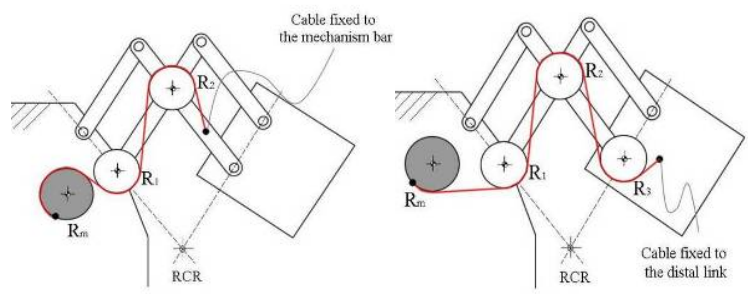

Fig. 7. Examples of the Patented principle for the actuation of RCM employing: two idle pulleys (left) and three idle pulleys (right).

The speed reduction ratio can be computed as:

$$
r=\frac{R_{m}}{\sum_{i=1}^{n} R_{i}}=\frac{R_{m}}{R_{e q}}
$$

where, $R_{i}$ is the radius of each i-esim idle pulley, $R_{m}$ is the radius of the driven pulley and $R_{e q}$ is the equivalent radius of a capstan that should be used for achieving the same speed reduction. Thus, the mechanisms can be seen as a capstan pulley system where the radius of the capstan $\left(R_{e q}\right)$ is equal to the sum of the radius of the idle pulleys, with the main advantage of having the global dimensions of the mechanism reduced.

The complete transmission system that includes the flexion and extension cables is represented for the joint $j_{H 2}$ in Fig. 8. Reddotted line represents the path of the flexion cable and the bluesolid line the extension one. The cables are routed on two parallel paths with an offset of $0.8 \mathrm{~mm}$ thanks to a double slot applied on each pulley. All the pulleys are idle and the cables are only fixed at the motor pulley and at the link $L_{2}$ through a crimp-slot attachment (indicated in the picture as "attachment points").

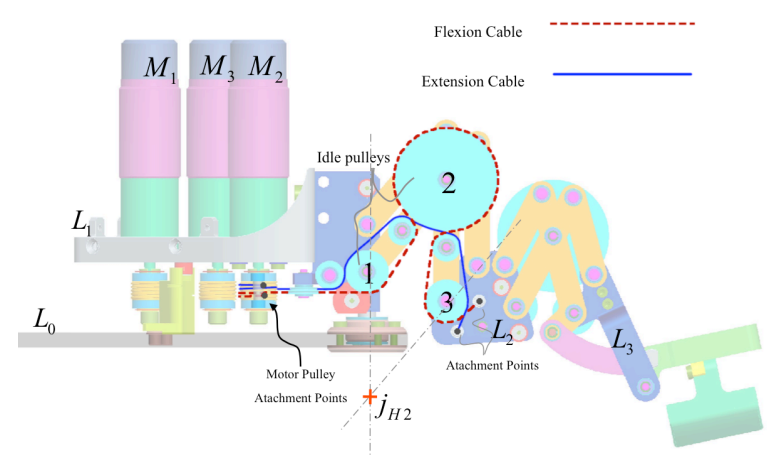

Fig. 8. Scheme of the cable-transmission routing for the actuation of joint $j_{H 2}$. cable is represented in blue continuous line while flexion cable in red-dotted line.

As previously anticipated the interesting feature of this kind of transmission is the implementation of an intrinsic speed reduction ratio between the rotation of the pulley of the motor $M_{2}$ and the joint $j_{H 2}$. The ratio $r_{22}$ can be easily calculated through the equation (1) considering the three idle pulleys 1,2 and 3 and the motor pulley $R_{m}$. The choice of dimensions was driven by trying to maximize the speed reduction ration considering the encumbrance limitations. This leads a total reduction ratio of approximately $1: 5$.

The transmission for the joint $j_{H 3}$ is located on the opposite side of the assembly (see Fig. 9). In this case, scheme is more complex since the cables have to go through the joint $j_{H 2}$. The cables run along the same path of the first transmission through the joint $j_{H 2}$ and follow an analogous routing scheme for the joint $j_{H 3}$.

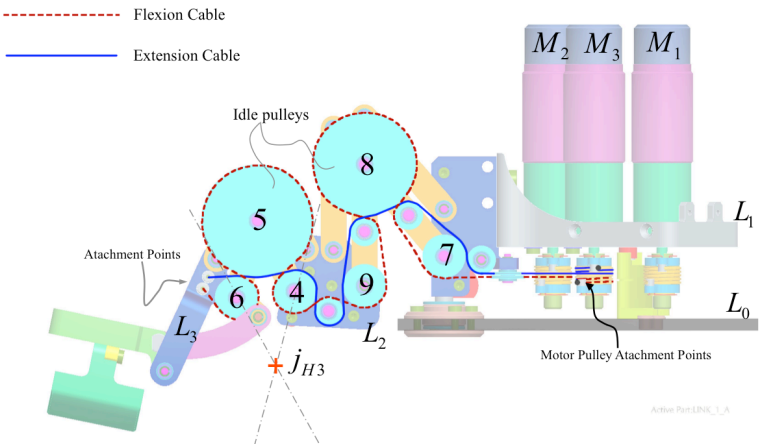

Fig. 9. Scheme of the transmission routing for the joint $j_{H 3}$ Extension cable is represented in blue continuous line while flexion cable in red-dotted line.

The speed reduction $r_{33}$ is computed with the equation (1) considering the radius of the pulleys 4,5 and 6 in Fig. 9. It is also important to consider that the rotation of the motor $M_{3}$ is also function of the rotation of the joint $j_{H 2}$. The ratio between the rotation of the join $j_{H_{2}}$ and the rotation of pulley of the motor $M_{3}$ when the joint $j_{H 3}$ is blocked is indicated with $r_{23}$ and can be computed with the formula (1) considering the radius of the pulley 7,8 and 9 .

The relation between the joints velocities and the motor axes velocities can be expressed as

$$
\dot{\theta}_{j}=\left[\begin{array}{ccc}
r_{11} & 0 & 0 \\
0 & r_{22} & 0 \\
0 & \frac{r_{33} r_{22}}{r_{23}} & r_{33} \\
0 & \gamma \frac{r_{33} r_{22}}{r_{23}} & \gamma r_{33}
\end{array}\right] \dot{\theta}_{m}=\mathbf{T} \dot{\theta}_{m}
$$

where $\dot{\theta}_{m}$ is the vector of the rotation speed of the motor pulleys, $\dot{\theta}_{j}$ is the vector of the rotation speed of the joints and $\gamma$ is the coupling ratio between the joints $j_{H 4}$ and $j_{H 3}$

The introduction of a speed reduction ratio that is intrinsically implemented by the transmission system has two main advantages: (a) for an assigned maximum force at the endeffector, the size of the motors can be reduced consequently reducing weight and encumbrance; (b) the stiffness of the transmission is increased since cables are loaded with a fraction of the tension. Moreover, as it is shown in [31], the resulting triangular form for the transmission matrix of equation (2) allows a further reduction of the actuators requirements. 
The actuators have been chosen consequently in order to achieve the identified maximum continuous performances. Three Minimotor 1727 equipped with low backlash planetary speed reducer with a ratio 14:1 (Minimotor 16/7) have been chosen. These motors are able to produce a maximum continuous torque before the speed reducer of $5 \mathrm{mNm}$ with peak velocity of 7800 rpm.

The final designed HE is able to exert a continuous net force of $5 \mathrm{~N}$ at the fingertip, weighting $0.51 \mathrm{~kg}$ for each finger that makes the system a quite high-performance robotic device with a ratio between continuous force versus self-weight very close to one. Such global weight of $1.1 \mathrm{~kg}$ is sustainable by the user for short periods but can cause fatigue after several minutes of use. For this reason the developed HE has been coupled with a 6-DoF mechanical tracker able to compensate the weight of the device [22]. The maximum joint velocity is approximately $11.6 \mathrm{rad} / \mathrm{s}$ when no load is applied and $5.6 \mathrm{rad} / \mathrm{s}$ at full load. In both cases the speed is more than sufficient to track fingers during normal movements. In Table 1 the main mechanical performances of the developed prototype of the HE are summarized.

Table 1: Mechanical Performances of the prototype of HE.

\begin{tabular}{ccc}
\hline Symbol & Quantity & Value \\
\hline DoF & Degrees of Freedom for each finger & 3 \\
$F_{\max }$ & Maximum continuous force & $5 \mathrm{~N}$ \\
$W$ & Weight of the whole device & $1.1 \mathrm{~kg}$ \\
$w_{a}$ & Weight of one finger mechanism & $0.51 \mathrm{~kg}$ \\
$\theta_{J \max }$ & Maximum Joint Velocity & $11 \mathrm{rad} / \mathrm{s}$ \\
\hline
\end{tabular}

\section{Force sensing}

A further action for improving the force accuracy and resolution has been taken by introducing two 3-DoF force sensors at the fingertips. This allows to strongly improve the performances of the devices through the design of force based control algorithms [33].

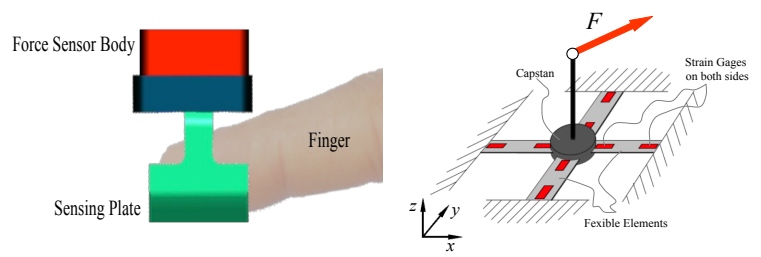

Fig. 10. Scheme of the positioning of the force sensor (left) and scheme of the Maltese Cross structure of the force sensor (right).

Each sensor is attached on the last link of the HE and the sensor spring is located on the dorsal side of the fingertip (see Fig. 10left) and the input plate (or sensing plate), i.e. the plate where the force is sensed, is held through a portal like structure under the fingertip. The conditioning electronics is integrated inside the box of the force sensor (see Fig.11-right). The signals are filtered at $500 \mathrm{~Hz}$ with a simple RC low-pass filter and the conditioning is achieved through a couple of precision operation al amplifier with a gain of 1000 (Burr Brown INA2128 by Texas Instruments ${ }^{\circledR}$ ).

The sensing principle is based on a Maltese-Cross spring represented in Fig. 10-right. This spring is composed by four beams disposed to form a cross. The beams converge in a central plate (capstan) where the forces are applied. Such structure was chosen because its spatial encumbrance is very limited and particularly suitable for the available spaces. Strain gauges are located on both the sides of the beams close to their ends. A complete bridge of strain gauges is used for each beam. The four gauge-signals are combined through least square fitting to estimate the forces over the three orthogonal directions. Details on the design and construction of the integrated force sensor are described in [17]. The main characteristics are summarized in Table 2 .

Table 2: Main performance of the developed sensors.

\begin{tabular}{cccc}
\hline Parameter & Value & Unit & $\%\left(\mathbf{f s}^{*}\right)$ \\
\hline Measuring Range & $>5$ & $\mathrm{~N}$ & $\mathrm{NA}$ \\
Linearity Error & $>2.7$ & $\mathrm{mN}$ & $0.06 \%$ \\
Hysteresis & $>10$ & $\mathrm{mN}$ & $0.21 \%$ \\
Resolution $* *$ & 2.0 & $\mathrm{mN}$ & $0.04 \%$ \\
* percentage of full scale value. \\
**Resolution is mainly limited by noise; for characterization, a high \\
precision low noise power supply has been employed which shows peak to \\
peak noise $V_{p-p} \leq 0.3 \mathrm{mV}$.
\end{tabular}

\section{Electronics}

A common solution for control-electronics of haptic devices is to employ off the shelf boards arranged in external boxes. This is the most reasonable solution for desktop devices especially when no force sensors are employed.

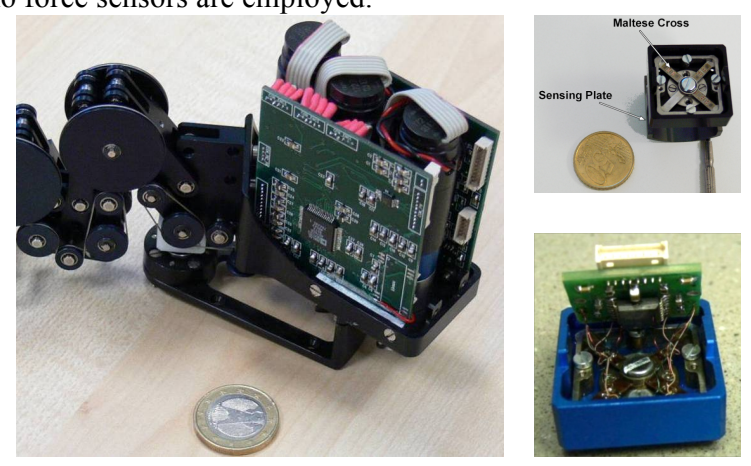

Fig. 11. Picture of the final PCBs of acquisition, driving electronics integrated (left) and conditioning (right).

For portable devices equipped with high precision force sensors, the design of the electronics and the cabling has to be carefully treated because: (a) it must be compatible with portability requirements and (b) must take into account electrical noise issues. For these reasons, custom electronic boards that are integrated in the mechanics have been purposely developed for the new HE including:

- One conditioning electronic board for each finger that is directly integrated in the housing of the force sensor. A instrumentation operational amplifier INA 2128 by Texas Instruments $^{\circledR}$ with balanced output is here employed (see Fig. 11-right);

- Two boards integrated in the motor housing (see Fig. 11 left). The first board includes a micro controller, a 4channels 16-bits $\mathrm{AD}$ for sensor signals (Analog Device AD7656), a 3-channels, 16 bits DA conversion chip for 
current driving, 3 encoders counters, a transceiver for standard RS-232 serial communication. The second board is equipped with current-drivers providing voltage-driven currents on 3 different channels for the control of the motor torque

The electronic boards are deeply integrated into the mechanical design of the device as shown in Fig. 11-left. This architecture guarantees low noise on force signals having shortlength cables for analog signals. Moreover, a strong simplification of the electrical cabling towards the main control-PC is achieved. A special care has been taken for minimizing the noise for analogue signals from sensors. Star-grounding scheme have been studied for limiting the noise on different grounding references and linear current driver have been chosen for reducing the electromagnetic noise.

\section{Tests and Characterization}

In this section we describe the procedures and the results obtained form a set of tests that have been conducted to characterize the main electro-mechanical properties of the developed HE-prototype and preliminary functional tests with users. All the experiments have been conducted on both the fingers of the HE showing very minor differences. For the sake of brevity we report only tests and experiment on one of the two fingers i.e. index finger.

\subsection{Mechanical Stiffness}

In order to estimate the mechanical stiffness of the device a measure has been done in the worst load condition. The Index HE has been mounted on a rigid plate that was solidly connected to ground. The end-effector was fixed to ground through a spherical joint in correspondence of the sensible point of the force sensor. The force was then increased up to the maximum $(+5 \mathrm{~N})$ and lowered back to the minimum $(-5 N)$. The force was applied in a quasi-static way (load cycle of 40s). Rotations of the encoder and end-effector force have been recorded for several cycles. A minor hysteretic/non-linear behavior is observed due to the effects of bearing/speed reducer backlash, cable friction and force sensor nonlinearity.

The stiffness in the worst direction resulted approximately $k_{s}=1[\mathrm{~N} / \mathrm{mm}]$, value that is in line with the performances of common commercial desktop haptic interfaces.

\subsection{Dynamic parameters identification}

Modeling and identification of main dynamic properties of the developed HE is achieved through a set of experimental tests. Each finger of the $\mathrm{HE}$ can be modeled as a Multiple Input Multiple Output (MIMO) system characterized by a 3 by 3 matrix transfer function with having the three motor torque as input the three motor position as outputs. However, general performance assessment of the device can be done on simplified SISO (Single Input Single Output) model based on the evaluation of worst-case conditions. Worst-case is considered the most critical position and the most critical direction in the workspace in which the bandwidth is limited at most. The aim is to provide a lower boundary of the bandwidth of the system.

On the base of estimations from FEM analysis and simplified dynamic models the worst-case has been identified as the adabduction movement with the finger fully extended. For this displacement only the first motor of the HE moves while the second and third are fixed against the end-stop.

The experimental characterization has been conducted in this position, commanding the first motor with a chirp current of fixed amplitude in a frequency range of $0-120 \mathrm{~Hz}$. Different amplitudes have been imposed in order to verify the linearity of the system. Fig. 12-top shows the Bode plot of the transfer function $G_{u L 0}=\theta_{1 m} / \tau_{1 m}$. where $\theta_{1 m}$ is the angular position first motor and $\tau_{1 m}$ is the torque exerted by the first motor. The system can be basically schematized as a 2-mass model with anti-resonant and resonant frequencies respectively of $f_{\text {ar }} \cong 23 \mathrm{~Hz} ; f_{r} \cong 29 \mathrm{~Hz}$.

The 2-mass system parameters, including equivalent mass of motor $\left(M_{m}\right)$, equivalent mass of joint $\left(M_{j}\right)$, motor viscosity $\left(C_{m}\right)$ and joint viscosity $\left(C_{j}\right)$ can be expressed in the reference frame of the end-effector: $M_{m}=0.29 \mathrm{~kg}, M_{j}=0.15 \mathrm{~kg}, C_{m}=7.72 \mathrm{Ns} / \mathrm{m}, C_{j}=$ $1.16 \mathrm{Ns} / \mathrm{m}$
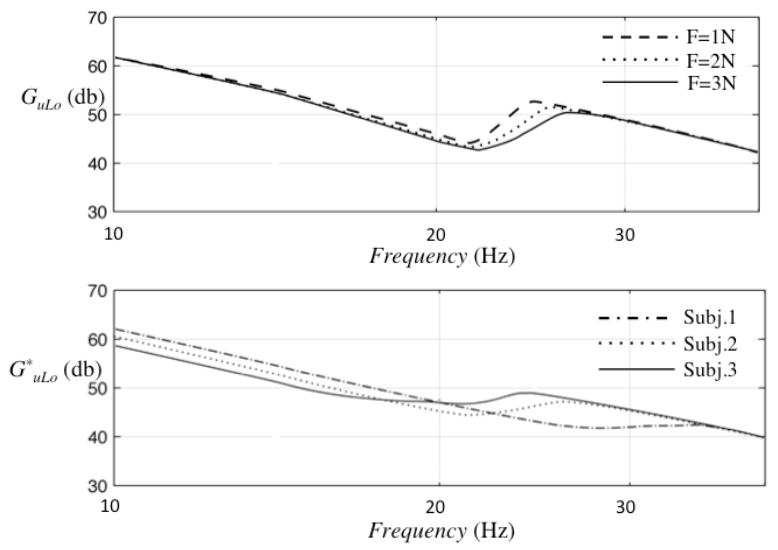

Fig. 12. On the top: Bode plot of the transfer function $G_{u L o}$ for different intensity of the chirp signals. On the bottom: Bode plot of the transfer function $G^{*}{ }_{u L o}$ with three different human finger in contact with the end-effector.

The same tests were done while the index finger is in contact with the plate exerting on it a constant force of $2 \mathrm{~N}$. The tests were done involving five different subjects that were asked to try to offer the maximum resistance by stiffening their finger. Fig 12bottom shows the magnitude Bode plots of the obtained transfer function $\left(G_{u L o}^{*}\right)$ for three different subjects. It can be observed that the human load introduce slight changes in the resonant and anti-resonant frequencies respect to the unloaded condition. Thus human finger impedance can be considered, in first instance, neglectable with respect to the device impedance and effects on $G_{u L o}$ are minor. Taking into account the observed variability, the resonant and anti-resonant frequencies are estimated in the range of $f_{a r} \cong 18 \div 23 \mathrm{~Hz}$ and $f_{r} \cong 25 \div 35 \mathrm{~Hz}$.

\subsection{Electronics: noise test}

Noise level on signals from force sensors is a critical issue that needs to be minimized in order to guarantee high accuracy of the device. Noise on force sensor signal is actively employed in the force control-loop and can affect the accuracy and resolution of the output force of the device. In order to estimate the noise for the realized electronic we perform a simple test acquiring the sensor signal and collecting the data for the estimation of the 
average noise on the force signal. The acquisition was done under the following conditions: (a) no load was applied in the force sensor; (b) the motors were powered; (c) the hand exoskeleton was fixed on an isolated support free of mechanical vibrations.

The average noise on the voltage readings resulted $N_{\sigma}=0.6[\mathrm{mV}]$ with peak-to-peak amplitude of $N_{p-p}=2.2[\mathrm{mV}]$, that corresponds to a maximum force resolution of $F_{r}=0.8[\mathrm{mN}]$.

\subsection{Preliminary tests on users}

With the aim of quantitatively verifying the performance of the device a set of preliminary tests with users have been conducted. The scope of such tests was not to provide an exact estimation of the maximum performances that can be achieved, but rather to provide an approximated quantitative measure of achievable force resolution during the use of the HE. An in-depth investigation of the optimization of control and relative performances is foreseen, but it is beyond the scope of this work.

Tests have been conducted using one finger of the hand exoskeleton. The proximal link of the hand exoskeleton, i.e., the dorsal plate, has been grounded in order to avoid possible disturbances introduced by unwanted displacement of the hand.
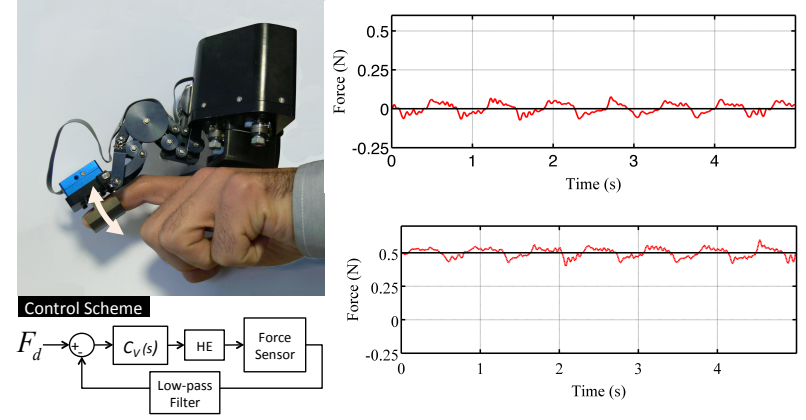

Fig. 13. Setup for tests with users (left) and preliminary results showing the force tracking accuracy during the use of the system.

The test consisted in performing the cyclical task of flexing the index finger in a natural way starting with the finger in a relaxed extended position (see Figure 13-left). The testers were asked to flex the index finger and invert the motion as soon as the fingertip has rotated approximately of $15^{\circ}-20^{\circ}$. No particular measures have been taken for controlling the accuracy of the rotation as its magnitude was not relevant. The testers were asked to repeat cyclically the movement with a frequency of approximately 1.5 $\mathrm{Hz}$. An audio pulsating signal was presented during the test to help to keep the frequency of the movement cycle roughly constant.

The employed algorithm is a simple closed-loop force controller with force compensation loop (Fig.13-bottom). The feedback signal from the force sensor is filtered through a lowpass filter with cut-off frequency set at $25 \mathrm{~Hz}$. The force error drives a compensation through a velocity control loop $\left(C_{v(s)}\right)$. High values for the gain of the closed loop improve the performance of the system but there are limitations due to stability of the system. The gain has been increased and set up to $90 \%$ of the stability limit. A null desired force $\left(F_{d 0}=0 \mathrm{~N}\right)$ and a constant force $\left(F_{d l}=\right.$ $500 \mathrm{mN}$ ) have been commanded and the residual uncompensated force that is delivered to the fingertip was measured through the force sensor. A plot of the results is represented in Figure 11-left showing the force deviation from the desired value (z-component of the sensor force is plotted). An average residual force error with a magnitude of $85-90 \mathrm{mN}$ is recorded for both the conditions.

Advanced types of controller including PID, Kalman filters, or position/velocity-based force controller will be studied for further improving the global performances but this is beyond the scope of this work.

\section{Conclusions}

This paper presents the electro-mechanical design and the experimental identification of main static and dynamic properties of a novel haptic Hand Exoskeleton (HE). The device is designed for virtual reality applications and it is particularly addressed to the artificial generation of forces that are felt during precision grasping of objects and surface exploration. The realized device is able to deliver three-degrees-of-freedom forces on the thumb and on the index fingertips of the user. The mechanical and electronic design of the device aims at maximizing the force accuracy by minimizing the effect of friction and inertial forces. A special patented force transmission system allows to achieve high force capabilities with reduced weight and encumbrances of the assembly. On-board force sensing at the level of the fingertip of the user allows introducing a controller able to compensate disturbing friction and inertia forces.

The design work leads to the realization of a prototype of the HE able to exert forces up to $5 \mathrm{~N}$ on each finger. The global weight of the device is $1.1 \mathrm{~kg}$ ( $0.51 \mathrm{~kg}$ for one finger structure). Identification of the electro-mechanical performances in worstcase condition and reported. The tests show that the device has mechanical performances in line with the design guidelines and specifications. Preliminary functional tests employing simple control strategy have been conducted showing an achievable force accuracy of approximately $85-90 \mathrm{mN}$. It is foreseen to achieve improvements of such performances with the implementation of more advanced control algorithms that will be studied in the next future

\section{References}

[1] Zarudiansky, A., 1981 "Remote handling devices,". United States Patents Archive, Pat. N. 4302138.

[2] Jau, B.M., 1988, "Anthropomorphic exoskeleton dual arm/hand telerobot controller," Proceedings of IEEE International Workshop on Intelligent Robots, pp.715-718, 1988.

[3] Burdea, G., Zhuang, J., Roskos, E., Silver, D., and Langrana N., 1992. "A portable dextrous master with force feedback," Presence: Teleoperators and Virtual Environments, l (1), pp. 18-28.

[4] Bergamasco, M., 1992, "Design of hand force feedback systems for glove-like advanced interfaces," Proceedings of IEEE International Workshop on Robot and Human Communication, pp. 286-293.

[5] Koyama, T., Yamano, I., Takemura, K., and Maeno, T., 2002, "Multi-fingered exoskeleton haptic device using passive force feedback for dexterous teleoperation," Proceedings of IEEE/RSJ International Conference on Intelligent Robots and Systems, Vol.3, pp. 2905-2910.

[6] Frisoli, A., Simoncini, F., Bergamasco, M., and Salsedo, F., 2007, "Kinematic Design of a Two Contact Points Haptic 
Interface for the Thumb and Index Fingers of the Hand," Journal of Mechanical Design, 129(5), pp. 520-529.

[7] Nakagawara, S., and Kajimoto, H., 2005, "An Encounter Type Multi Fingered Master Hand," Proceedings IEEE International Conference on Robotics and Automation, pp. 2667-2672.

[8] Gomez, D., Burdea, G., and Langrana, N., 1995, "Integration of the Rutgers Master II in a virtual reality simulation," Proceedings of IEEE Virtual Reality Annual International Symposium, pp. 198-202.

[9] Wege, A., Kondak, K., and Hommel, G., 2005, "Mechanical design and motion control of a hand exoskeleton for rehabilitation," Proceedings of IEEE International Conference on Mechatronics \& Automation, pp. 155-159.

[10] Mulas, M., Folgheraiter, M., and Gini, G., 2005 “An EMGcontrolled exoskeleton for hand rehabilitation," Proceedings of International Conference on Rehabilitation Robotics, pp. 371-374.

[11] Ito, S., Kawasaki, H., Ishigure, Y., Natsume, M., Mouri, T., and Nishimoto, Y., 2011, "A design of fine motion assist equipment for disabled hand in robotic rehabilitation system," Journal of the Franklin Institute, 348(1), pp. 79-89.

[12] Brown, P., Jones, D., Singh, S. K., and Rosen, J. M., 1993, "The exoskeleton glove for control of paralyzed hands," Proceedings of the IEEE International Conference on Robotics and Automation, pp. 642-647 vol.1.

[13] Watanabe, K., and Morishita, H., 2007, "A Prototype of Index-Finger PIP Joint Motion Amplifier for Assisting Patients with Impaired Hand Mobility," Proceedings IEEE International Conference on Robotics and Automation, pp. 10-14.

[14] Sarakoglou, I., Tsagarakis, N. G., and Caldwell, D. G., 2004, "Occupational and physical therapy using a hand exoskeleton based exerciser," Proceedings of the IEEE/RSJ International Conference on Intelligent Robots and Systems, pp. 29732978.

[15] Shields, B.L., Main, J.A., Peterson, S.W., Strauss, A.M., 1997, "An anthropomorphic hand exoskeleton to prevent astronaut hand fatigue during extravehicular activities," IEEE Transactions on Systems, Man and Cybernetics, Part A, 27 (5), pp.668-673.

[16] Yamada, Y., Morizono, T., Sato, S., Shimohira, T., Umetani, Y., Yoshida, T., and Aoki, S., 2001, "Proposal of a SkilMate finger for EVA gloves," Proceedings of the IEEE International Conference on Robotics and Automation, pp. 1406-1412, vol.2.

[17] Fontana, M., Marcheschi, S., Salsedo, F., and Bergamasco, M., 2012, "A three-axis force sensor for dual finger haptic interfaces," Sensors (Switzerland), 12(10), pp. 13598-13616.

[18] Jones, L.A., and Lederman, S.J., 2006, Human Hand Function; Oxford University Press: New York, NY, USA.

[19] Lee, J.W., Rim, K., 1990, "Maximum finger force prediction using a planar simulation of the middle finger," Proceedings of Institution of Mechanical Engineers, 204, pp. 169-178.

[20] Levin, S., Pearsall, G., Ruderman, R.J., 1978, "Von Frey's method of measuring pressure sensibility in the hand: An engineering analysis of the Weinstein-Semmes pressure aesthesiometer," Journal of Hand Surgery, 3, 211-216.

[21] Redmond, R., Aina, R., Gorti, T., Hannaford, B., 2010 "Haptic Characteristics of Some Activities of Daily Living," Proceedings of Haptics Symposium, pp. 71-76.

[22] Tarri, F., Fontana, M., Salsedo, F., Marcheschi, S., and Bergamasco, M., 2009, "Modular weight-balanced mechanical tracker for portable haptics," Proceedings - IEEE International Conference on Robotics and Automation, pp. 1473-1478.

[23] Springer, S., and Ferrier, N.J., 1999 "Design and control of a force-reflecting haptic interface for teleoperational grasping", ASME Journal of Mechanical Design, 124 (2) pp. 277-283.

[24] Chang, L. Y., and Matsuoka, Y., 2006, "A kinematic thumb model for the ACT hand," Proceedings - IEEE International Conference on Robotics and Automation, pp. 1000-1005.

[25] Leijnse, J., Quesada, P. M., and Spoor, C. W., 2010 "Kinematic evaluation of the finger's interphalangeal joints coupling mechanism-variability, flexionextension differences, triggers, locking swanneck deformities, anthropometric correlations," Journal of Biomechanics, 43 (12), pp. 2381-2393.

[26] Pheasant, S., and Haslegrave, C. M., 2006 Anthropometry, ergonomics, and the design of work. Taylor \& Francis, 2006

[27] Avizzano, C. A., Barbagli, F., Frisoli, A., Bergamasco, M., 2000 "The Hand Force Feedback: Analysis and Control of a Haptic Device for the Human Hand", Proceedings of the International Conference on Systems, Man and Cybernetics, pp. 98-103.

[28] Pei, X., Yu, J., Bi, S., and Zong, G., 2007, "Enumeration and Type Synthesis of One-DOF Remote-Center-of-Motion Mechanisms" Proceedings of the International Federation for the Promotion of Mechanism and Machine Science Congress, pp.18-21.

[29] Fiaschi, M., Salsedo, F., Bergamasco, M., "Analysis and Design of an Hand Exoskeleton", Bachelor Thesis in Mechanical Engineer, University of Pisa, 2003.

[30] Salsedo, F., Marcheschi, S., Fontana, M., and Bergamasco, M., 2011, "Tactile transducer based on electromechanical solenoids," 2011 IEEE World Haptics Conference, WHC 2011, pp. 581-586.

[31] Fontana, M., Dettori, A., Salsedo, F., and Bergamasco, M., 2009, "Mechanical design of a novel hand exoskeleton for accurate force displaying," Proceedings - IEEE International Conference on Robotics and Automation, pp. 1704-1709.

[32] Bergamasco, M., Salsedo, F., and Fontana, M., 2009 "Method for remote mechanism actuation and exoskeleton haptic interface based thereon," European Patent, Pat. N. EP2337656 A1.

[33] Marcheschi, S., Salsedo, F., Fontana, M., Tarri, F., PortilloRodríguez, O., and Bergamasco, M., 2007, "High performance explicit force control for finger interaction haptic interface," Proceedings of Wordhaptics 2007,pp. 464 469. 